IJMMS 32:10 (2002) 627-633

PII. S0161171202110155

http://ijmms.hindawi.com

(c) Hindawi Publishing Corp.

\title{
A NEW PROOF OF SOME IDENTITIES OF BRESSOUD
}

\section{ROBIN CHAPMAN}

Received 18 October 2001

We provide a new proof of the following two identities due to Bressoud: $\sum_{m=0}^{N} q^{m^{2}}\left[\begin{array}{c}N \\ m\end{array}\right]=$ $\sum_{m=-\infty}^{\infty}(-1)^{m} q^{m(5 m+1) / 2}\left[\begin{array}{c}2 N \\ N+2 m\end{array}\right], \sum_{m=0}^{N} q^{m^{2}+m}\left[\begin{array}{c}N \\ m\end{array}\right]=\left(1 /\left(1-q^{N+1}\right)\right) \sum_{m=-\infty}^{\infty}(-1)^{m} \times$ $q^{m(5 m+3) / 2}\left[\begin{array}{c}2 N+2 \\ N+2 m+2\end{array}\right]$, which can be considered as finite versions of the Rogers-Ramanujan identities.

2000 Mathematics Subject Classification: 05A19.

In [1], Bressoud proves the following theorem, from which the Rogers-Ramanujan identities follow on letting $N \rightarrow \infty$.

THEOREM 1. For each integer $N \geq 0$,

$$
\begin{aligned}
\sum_{m=0}^{N} q^{m^{2}}\left[\begin{array}{l}
N \\
m
\end{array}\right] & =\sum_{m=-\infty}^{\infty}(-1)^{m} q^{m(5 m+1) / 2}\left[\begin{array}{c}
2 N \\
N+2 m
\end{array}\right], \\
\sum_{m=0}^{N} q^{m^{2}+m}\left[\begin{array}{l}
N \\
m
\end{array}\right] & =\frac{1}{1-q^{N+1}} \sum_{m=-\infty}^{\infty}(-1)^{m} q^{m(5 m+3) / 2}\left[\begin{array}{c}
2 N+2 \\
N+2 m+2
\end{array}\right] .
\end{aligned}
$$

Here,

$$
\left[\begin{array}{l}
N \\
m
\end{array}\right]= \begin{cases}\frac{(q)_{N}}{(q)_{m}(q)_{N-m}} & \text { if } 0 \leq m \leq N \\
0 & \text { otherwise }\end{cases}
$$

denotes a Gaussian binomial coefficient, where we adopt the standard $q$-series notation

$$
(q)_{n}=\prod_{j=1}^{n}\left(1-q^{j}\right) .
$$

We give an alternative proof of Theorem 1 by showing that the left and right sides of (1) satisfy the same recurrence relations.

Define, for integers $a$ and $N \geq 0$,

$$
S_{a}(N)=\sum_{n=0}^{N} q^{n^{2}+a n}\left[\begin{array}{l}
N \\
n
\end{array}\right] .
$$

LEMMA 2. For each integer $N \geq 1$ and each $a$,

$$
\begin{aligned}
& S_{a}(N)=S_{a}(N-1)+q^{N+a} S_{a+1}(N-1), \\
& S_{a}(N)=S_{a+1}(N-1)+q^{a+1} S_{a+2}(N-1) .
\end{aligned}
$$


Proof. Using the identity

$$
\left[\begin{array}{l}
N \\
n
\end{array}\right]=q^{N-n}\left[\begin{array}{l}
N-1 \\
n-1
\end{array}\right]+\left[\begin{array}{c}
N-1 \\
n
\end{array}\right]
$$

gives

$$
\begin{aligned}
S_{a}(N) & =q^{N} \sum_{n=1}^{N} q^{n^{2}+(a-1) n}\left[\begin{array}{c}
N-1 \\
n-1
\end{array}\right]+\sum_{n=0}^{N-1} q^{n^{2}+a n}\left[\begin{array}{c}
N-1 \\
n
\end{array}\right] \\
& =q^{N} \sum_{n=0}^{N-1} q^{(n+1)^{2}+(a-1)(n+1)}\left[\begin{array}{c}
N-1 \\
n
\end{array}\right]+S_{a}(N-1) \\
& =q^{N+a} S_{a+1}(N-1)+S_{a}(N-1) .
\end{aligned}
$$

On the other hand, using the identity

$$
\left[\begin{array}{l}
N \\
n
\end{array}\right]=\left[\begin{array}{l}
N-1 \\
n-1
\end{array}\right]+q^{n}\left[\begin{array}{c}
N-1 \\
n
\end{array}\right]
$$

gives

$$
\begin{aligned}
S_{a}(N) & =\sum_{n=1}^{N} q^{n^{2}+a n}\left[\begin{array}{c}
N-1 \\
n-1
\end{array}\right]+\sum_{n=0}^{N-1} q^{n^{2}+(a+1) n}\left[\begin{array}{c}
N-1 \\
n
\end{array}\right] \\
& =\sum_{n=0}^{N-1} q^{(n+1)^{2}+a(n+1)}\left[\begin{array}{c}
N-1 \\
n
\end{array}\right]+S_{a+1}(N-1) \\
& =q^{a+1} S_{a+2}(N-1)+S_{a+1}(N-1) .
\end{aligned}
$$

We now equate (5) and (6).

LEMMA 3. For integers $N \geq 0$ and each $a$,

$$
S_{a}(N)+\left(q^{N+a+1}-1\right) S_{a+1}(N)-q^{a+1} S_{a+2}(N)=0 .
$$

Proof. Equating (5) and (6) gives

$$
S_{a}(N-1)+\left(q^{N+a}-1\right) S_{a+1}(N-1)-q^{a+1} S_{a+2}(N-1)=0
$$

for $N \geq 1$. Replacing $N$ by $N+1$ gives

$$
S_{a}(N)+\left(q^{N+a+1}-1\right) S_{a+1}(N)-q^{a+1} S_{a+2}(N)=0 .
$$

We will use the $a=0$ case of Lemma 3 which is

$$
S_{0}(N)+\left(q^{N+1}-1\right) S_{1}(N)-q S_{2}(N)=0 .
$$

Clearly, $S_{a}(0)=1$ for all $a$. Also, for $N>0$, (5) gives

$$
S_{0}(N)=S_{0}(N-1)+q^{N} S_{1}(N-1)
$$


and, together with (14), gives

$$
\begin{aligned}
S_{1}(N) & =S_{1}(N-1)+q^{N+1} S_{2}(N-1) \\
& =S_{1}(N-1)+q^{N}\left[S_{0}(N-1)+\left(q^{N}-1\right) S_{1}(N-1)\right] \\
& =q^{N} S_{0}(N-1)+\left(q^{2 N}-q^{N}+1\right) S_{1}(N-1) .
\end{aligned}
$$

Together with the initial conditions $S_{0}(0)=S_{1}(0)=1$, (15) and (16) completely define $S_{0}(N)$ and $S_{1}(N)$ for $N \geq 0$.

We now gather some consequences of these recurrences which will be used later.

LEMMA 4. For $N \geq 2$,

$$
S_{0}(N)=\left(1+q^{2 N-1}\right) S_{0}(N-1)+q^{N}\left(1-q^{N}\right) S_{1}(N-2)
$$

and for $N \geq 1$,

$$
S_{1}(N)=q^{N} S_{0}(N)+\left(1-q^{N}\right) S_{1}(N-1) .
$$

Proof. First of all, from (15) and (16), we have

$$
S_{1}(N)-q^{N} S_{0}(N)=\left(1-q^{N}\right) S_{1}(N-1)
$$

and so, for $N \geq 2$,

$$
S_{1}(N-1)-q^{N-1} S_{0}(N-1)=\left(1-q^{N-1}\right) S_{1}(N-1) .
$$

Hence, by (15) again,

$$
\begin{aligned}
S_{0}(N) & =S_{0}(N-1)+q^{N} S_{1}(N-1) \\
& =S_{0}(N-1)+q^{N}\left[q^{N-1} S_{0}(N-1)+\left(1-q^{N}\right) S_{1}(N-2)\right] \\
& =\left(1+q^{2 N-1}\right) S_{0}(N-1)+q^{N}\left(1-q^{N}\right) S_{1}(N-2),
\end{aligned}
$$

and also by using (16),

$$
\begin{aligned}
S_{1}(N) & =q^{N} S_{0}(N-1)+\left(1-q^{N}+q^{2 N}\right) S_{1}(N-1) \\
& =q^{N}\left[S_{0}(N)-q^{N} S_{1}(N-1)\right]+\left(1-q^{N}+q^{2 N}\right) S_{1}(N-1) \\
& =q^{N} S_{0}(N)+\left(1-q^{N}\right) S_{1}(N-1) .
\end{aligned}
$$

The recurrences (17) and (18) with the initial conditions $S_{0}(0)=S_{1}(0)=1, S_{0}(1)=$ $1+q$ define $S_{0}(N)$ and $S_{1}(N)$ uniquely for $N \geq 0$.

Let

$$
\begin{aligned}
& B_{0}(N)=\sum_{m}(-1)^{m} q^{m(5 m+1) / 2}\left[\begin{array}{c}
2 N \\
N+2 m
\end{array}\right], \\
& B_{1}(N)=\sum_{m}(-1)^{m} q^{m(5 m+3) / 2}\left[\begin{array}{c}
2 N+2 \\
N+2 m+2
\end{array}\right]
\end{aligned}
$$


denote the sums appearing on the right sides of the identities in Theorem 1. Setting $r=N+2 m$ in the definition of $B_{0}(N)$ gives

$$
\begin{aligned}
B_{0}(N) & =\sum_{r \equiv N(4)} q^{(5 / 8)(r-N)^{2}+(1 / 4)(r-N)}\left[\begin{array}{c}
2 N \\
r
\end{array}\right]-\sum_{r \equiv N+2(4)} q^{(5 / 8)(r-N)^{2}+(1 / 4)(r-N)}\left[\begin{array}{c}
2 N \\
r
\end{array}\right] \\
& =q^{-1 / 40}\left[\sum_{r \equiv N(4)} q^{(5 / 8)(r-N+1 / 5)^{2}}\left[\begin{array}{c}
2 N \\
r
\end{array}\right]-\sum_{r \equiv N+2(4)} q^{(5 / 8)(r-N+1 / 5)^{2}}\left[\begin{array}{c}
2 N \\
r
\end{array}\right]\right] .
\end{aligned}
$$

This suggests the notation

$$
A(M, k, b)=\sum_{2 r \equiv M+k(8)} q^{(5 / 8)(r-M / 2+b)^{2}}\left[\begin{array}{c}
M \\
r
\end{array}\right]
$$

so that

$$
q^{1 / 40} B_{0}(N)=A\left(2 N, 0, \frac{1}{5}\right)-A\left(2 N, 4, \frac{1}{5}\right) .
$$

Of course, $A(M, k, b)=0$ if $M+k$ is odd, and $A(M, k, b)$ depends only on $M, b$ and the congruence class of $k$ modulo 8 . A similar computation yields

$$
q^{9 / 40} B_{1}(N)=A\left(2 N+2,2,-\frac{2}{5}\right)-A\left(2 N+2,-2,-\frac{2}{5}\right) .
$$

We aim at showing that $B_{0}(N)$ and $\left(1-q^{N+1}\right) B_{1}(N)$ satisfy the same system of recurrences as $S_{0}(N)$ and $S_{1}(N)$.

LEMMA 5. The following holds

$$
A(M, k, b)=A(M,-k,-b)
$$

for each $M, k$, and $b$.

Proof. Replacing $r$ by $M-r$ in the sum for $A(M, k, b)$ yields

$$
\begin{aligned}
A(M, k, b) & =\sum_{2 M-2 r \equiv M+k(8)} q^{(5 / 8)(M / 2-r+b)^{2}}\left[\begin{array}{c}
M \\
M-r
\end{array}\right] \\
& =\sum_{2 r \equiv M-k(8)} q^{(5 / 8)(r-M / 2-b)^{2}}\left[\begin{array}{c}
M \\
r
\end{array}\right] \\
& =A(M,-k,-b) .
\end{aligned}
$$

We now wish to produce recurrences for the $A(M, k, b)$.

LEMMA 6. The following holds

$$
\begin{aligned}
& A(M+1, k, b)=A\left(M, k-1, b+\frac{1}{2}\right)+q^{M / 2+1 / 10-b} A\left(M, k+1, b+\frac{3}{10}\right), \\
& A(M+1, k, b)=A\left(M, k+1, b-\frac{1}{2}\right)+q^{M / 2+1 / 10+b} A\left(M, k-1, b-\frac{3}{10}\right)
\end{aligned}
$$

for each $M, k$, and $b$. 
Proof. Using the formula

$$
\left[\begin{array}{c}
M+1 \\
r
\end{array}\right]=\left[\begin{array}{c}
M \\
r-1
\end{array}\right]+q^{r}\left[\begin{array}{c}
M \\
r
\end{array}\right]
$$

in the definition of $A(M+1, k, b)$ gives $A(M+1, k, b)=S_{1}+S_{2}$, where

$$
\begin{aligned}
S_{1} & =\sum_{2 r \equiv M+k+1(8)} q^{(5 / 8)(r-M / 2-1 / 2+b)^{2}}\left[\begin{array}{c}
M \\
r-1
\end{array}\right] \\
& =\sum_{2 s \equiv M+k-1(8)} q^{(5 / 8)(s-M / 2+1 / 2+b)^{2}}\left[\begin{array}{c}
M \\
s
\end{array}\right] \\
& =A\left(M, k-1, b+\frac{1}{2}\right), \\
S_{2} & =\sum_{2 r \equiv M+k+1(8)} q^{r+(5 / 8)(r-M / 2-1 / 2+b)^{2}}\left[\begin{array}{c}
M \\
r
\end{array}\right] .
\end{aligned}
$$

But

$$
r+\frac{5(r-M / 2-1 / 2+b)^{2}}{8}=\frac{5(r-M / 2+3 / 10+b)^{2}}{8}+\frac{M}{2}+\frac{1}{10}-b .
$$

Hence,

$$
A(M+1, k, b)=A\left(M, k-1, b+\frac{1}{2}\right)+q^{M / 2+1 / 10-b} A\left(M, k+1, b+\frac{3}{10}\right) .
$$

Consequently, by Lemma 5 also,

$$
\begin{aligned}
A(M+1, k, b) & =A(M+1,-k,-b) \\
& =A\left(M,-k-1,-b+\frac{1}{2}\right)+q^{M / 2+1 / 10+b} A\left(M,-k+1,-b+\frac{3}{10}\right) \\
& =A\left(M, k+1, b-\frac{1}{2}\right)+q^{M / 2+1 / 10+b} A\left(M, k-1, b-\frac{3}{10}\right) .
\end{aligned}
$$

It is convenient to note that replacing $M$ by $M-1$ in these identities gives

$$
\begin{aligned}
A(M, k, b) & =A\left(M-1, k-1, b+\frac{1}{2}\right)+q^{M / 2-2 / 5-b} A\left(M-1, k+1, b+\frac{3}{10}\right) \\
& =A\left(M-1, k+1, b-\frac{1}{2}\right)+q^{M / 2-2 / 5+b} A\left(M-1, k-1, b-\frac{3}{10}\right) .
\end{aligned}
$$

LEMMA 7. The sums $B_{0}(N)$ and $B_{1}(N)$ obey the recurrences

$$
B_{0}(N)=\left(1+q^{2 N-1}\right) B_{0}(N-1)+q^{N} B_{1}(N-2)
$$

for $N \geq 2$ and

$$
B_{1}(N)=\left(1-q^{N+1}\right) B_{1}(N-1)+q^{N}\left(1-q^{N+1}\right) B_{0}(N)
$$

for $N \geq 1$. 
Proof. We compute

$$
\begin{aligned}
A\left(2 N, k, \frac{1}{5}\right)= & A\left(2 N-1, k+1,-\frac{3}{10}\right)+q^{N-1 / 5} A\left(2 N-1, k-1,-\frac{1}{10}\right) \\
= & A\left(2 N-2, k, \frac{1}{5}\right)+q^{N-3 / 5} A(2 N-2, k+2,0) \\
& +q^{N-1 / 5} A\left(2 N-2, k-2, \frac{2}{5}\right)+q^{2 N-1} A\left(2 N-2, k, \frac{1}{5}\right) \\
= & \left(1+q^{2 N-1}\right) A\left(2 N-2, k, \frac{1}{5}\right)+q^{N-3 / 5} A(2 N-2, k+2,0) \\
& +q^{N-1 / 5} A\left(2 N-2, k-2, \frac{2}{5}\right) .
\end{aligned}
$$

In particular,

$$
\begin{aligned}
A\left(2 N, 0, \frac{1}{5}\right)= & \left(1+q^{2 N-1}\right) A\left(2 N-2,0, \frac{1}{5}\right) \\
& +q^{N-3 / 5} A(2 N-2,2,0)+q^{N-1 / 5} A\left(2 N-2,-2, \frac{2}{5}\right), \\
A\left(2 N, 4, \frac{1}{5}\right)= & \left(1+q^{2 N-1}\right) A\left(2 N-2,4, \frac{1}{5}\right) \\
& +q^{N-3 / 5} A(2 N-2,6,0)+q^{N-1 / 5} A\left(2 N-2,2, \frac{2}{5}\right) \\
& +q^{N-3 / 5} A(2 N-2,-2,0)+q^{N-1 / 5} A\left(2 N-2,2, \frac{2}{5}\right) .
\end{aligned}
$$

Noting that

$$
\begin{gathered}
A(2 N-2,2,0)=A(2 N-2,-2,0), \\
A\left(2 N-2,2, \frac{2}{5}\right)=A\left(2 N-2,-2,-\frac{2}{5}\right),
\end{gathered}
$$

subtracting gives

$$
\begin{aligned}
q^{1 / 40} B_{0}(N)= & A\left(2 N, 0, \frac{1}{5}\right)-A\left(2 N, 4, \frac{1}{5}\right) \\
= & \left(1+q^{2 N-1}\right)\left[A\left(2 N-2,0, \frac{1}{5}\right)-A\left(2 N-2,4, \frac{1}{5}\right)\right] \\
& +q^{N-1 / 5}\left[A\left(2 N-2,2,-\frac{2}{5}\right)-A\left(2 N-2,-2,-\frac{2}{5}\right)\right] \\
= & \left(1+q^{2 N-1}\right) q^{1 / 40} B_{0}(N-1)+q^{N-1 / 5} q^{9 / 40} B_{1}(N-2)
\end{aligned}
$$

and so

$$
B_{0}(N)=\left(1+q^{2 N-1}\right) B_{0}(N-1)+q^{N} B_{1}(N-2) .
$$


Also,

$$
\begin{aligned}
A\left(2 N+2, k,-\frac{2}{5}\right)= & A\left(2 N+1, k-1, \frac{1}{10}\right)+q^{N+1} A\left(2 N+1, k+1,-\frac{1}{10}\right) \\
= & A\left(2 N, k,-\frac{2}{5}\right)+q^{N+1 / 5} A\left(2 N, k-2,-\frac{1}{5}\right) \\
& +q^{N+1} A\left(2 N, k, \frac{2}{5}\right)+q^{2 N+6 / 5} A\left(2 N, k+2, \frac{1}{5}\right) \\
= & A\left(2 N, k,-\frac{2}{5}\right)+q^{N+1} A\left(2 N,-k,-\frac{2}{5}\right) \\
& +q^{N+1 / 5} A\left(2 N, 2-k, \frac{1}{5}\right)+q^{2 N+6 / 5} A\left(2 N, k+2, \frac{1}{5}\right) .
\end{aligned}
$$

Consequently,

$$
\begin{aligned}
q^{9 / 40} B_{1}(N)= & A\left(2 N+2,2,-\frac{2}{5}\right)-A\left(2 N+2,-2,-\frac{2}{5}\right) \\
= & A\left(2 N, 2,-\frac{2}{5}\right)+q^{N+1} A\left(2 N,-2-\frac{2}{5}\right) \\
& -A\left(2 N,-2,-\frac{2}{5}\right)-q^{N+1} A\left(2 N, 2,-\frac{2}{5}\right) \\
& +q^{N+1 / 5}\left[A\left(2 N, 0, \frac{1}{5}\right)-A\left(2 N, 4, \frac{1}{5}\right)\right] \\
& +q^{2 N+6 / 5}\left[A\left(2 N, 4, \frac{1}{5}\right)-A\left(2 N, 0, \frac{1}{5}\right)\right] \\
= & \left(1-q^{N+1}\right)\left[q^{9 / 40} B_{1}(N-1)+q^{N+1 / 5} q^{1 / 40} B_{0}(N)\right]
\end{aligned}
$$

and so

$$
B_{1}(N)=\left(1-q^{N+1}\right) B_{1}(N-1)+q^{N}\left(1-q^{N+1}\right) B_{0}(N) .
$$

By Lemma $4, S_{0}(N)$ and $\left(1-q^{N+1}\right) S_{1}(N)$ satisfy the same recurrences as $B_{0}(N)$ and $B_{1}(N)$. Also, $S_{0}(0)=1=B_{0}(0), S_{0}(1)=1+q=B_{0}(1)$, and $(1-q) S_{1}(0)=1-q=B_{1}(0)$. Consequently, we deduce Theorem 1: $S_{0}(N)=B_{0}(N)$ and $\left(1-q^{N+1}\right) S_{1}(N)=B_{1}(N)$.

\section{REFERENCES}

[1] D. M. Bressoud, Some identities for terminating q-series, Math. Proc. Cambridge Philos. Soc. 89 (1981), no. 2, 211-223.

Robin CHAPMAN: SCHOOL OF MATHEMATICAL SCIENCES, UNIVERSITY OF EXETER, EXETER, EX4 4QE, UK

E-mail address: rjc@maths.ex.ac.uk 


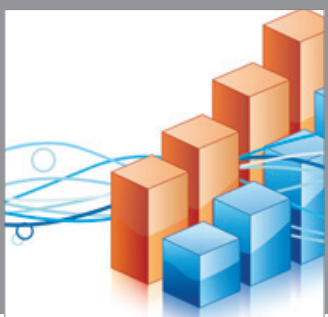

Advances in

Operations Research

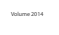

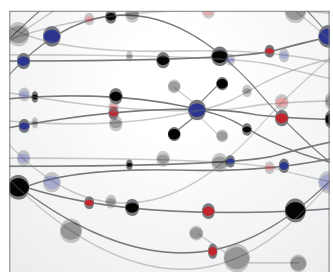

\section{The Scientific} World Journal
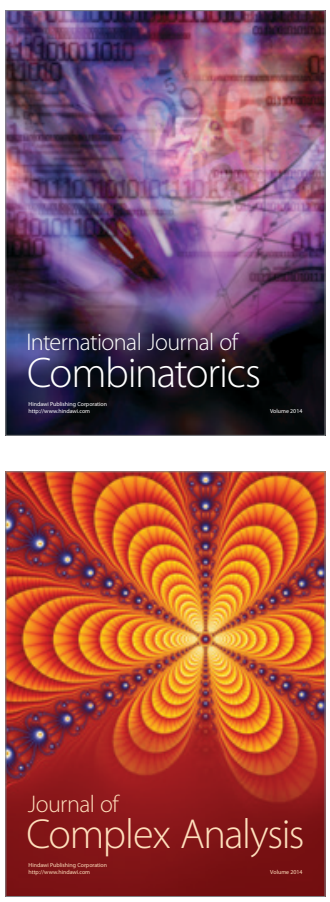

International Journal of

Mathematics and

Mathematical

Sciences
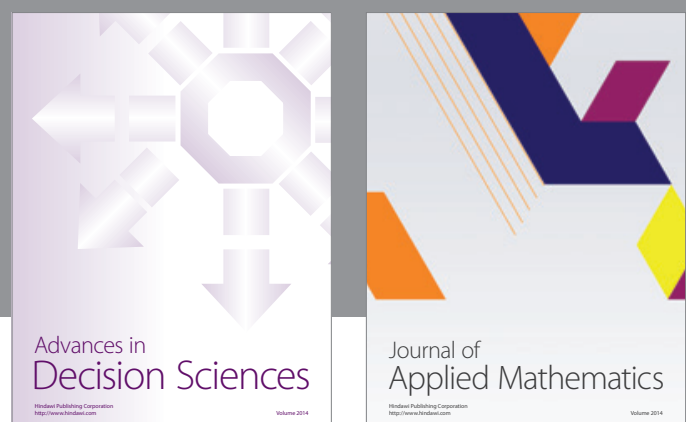

Journal of

Applied Mathematics
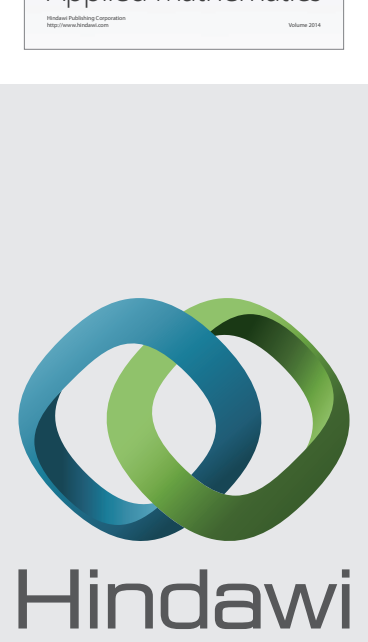

Submit your manuscripts at http://www.hindawi.com
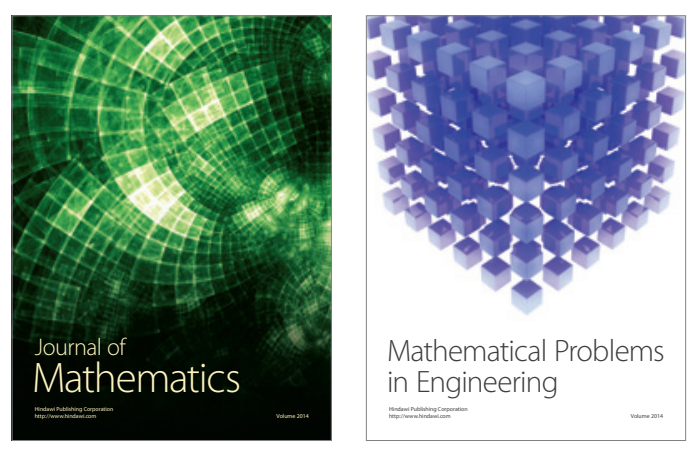

Mathematical Problems in Engineering
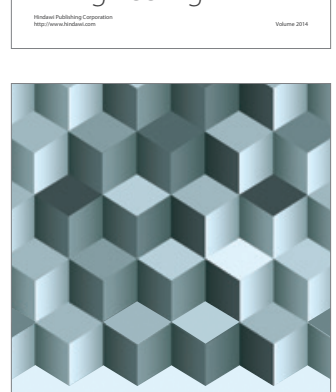

Journal of

Function Spaces
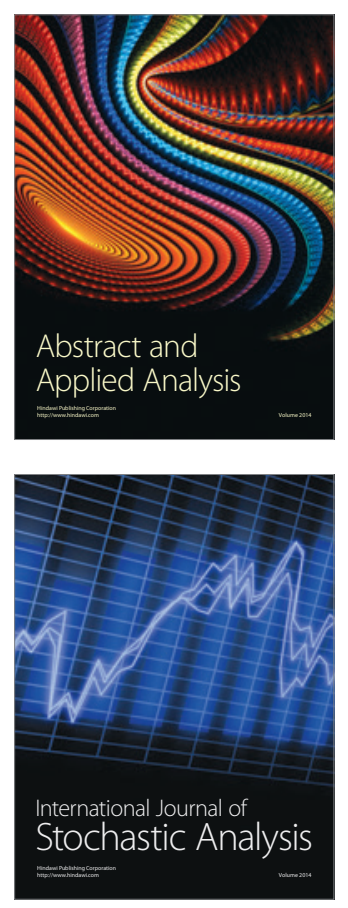

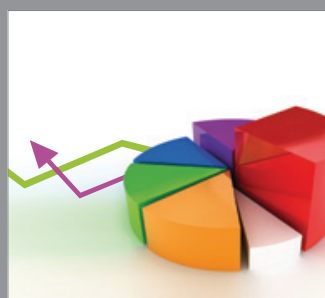

ournal of

Probability and Statistics

Promensencen
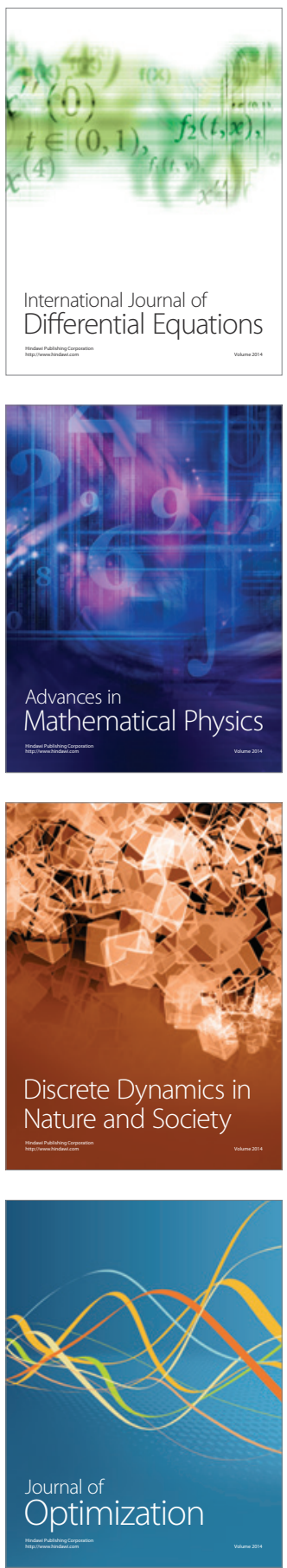